\title{
Monitoring source in an unconscious plagiarism paradigm
}

\author{
JOSHUA D. LANDAU and RICHARD L. MARSH \\ University of Georgia, Athens, Georgia
}

\begin{abstract}
Current laboratory paradigms used to assess unconscious plagiarism consist of three tasks. First, participants generate solutions to a puzzle task with a partner (initial generation task); second, they recall their individual contribution (recall-own task); and third, they attempt to create new solutions that were not offered previously (generate-new task). An analysis of these tasks indicated that they differ in terms of the source monitoring they require. The two generative tasks require less differentiated information (e.g., familiarity) and relatively lax decision criteria. The recall-own task, however, demands more differentiated information and more extended decision criteria. In two experiments, factors known to influence source monitoring were manipulated. Consistent with the analysis, no effects were associated with the generative tasks. Recall-own plagiarisms increased when self- and other-generated solutions were difficult to distinguish (Experiment 1) and decreased when the two sources were easier to distinguish (Experiment 2).
\end{abstract}

Offering an idea or a solution to a problem under the belief that it is one's novel contribution, when that idea or solution has actually been encountered earlier, constitutes cryptomnesia, or unconscious plagiarism (Taylor, 1965). Currently, two paradigms have been used successfully to study the laboratory analogue of unconscious plagiarism. The first, developed by Brown and Murphy (1989), assessed unconscious plagiarism in a category generation paradigm that consisted of three sequential tasks. The first task (called initial generation) required participants to take turns in groups of 4 , generating exemplars from four categories (e.g., musical instruments, sports, etc.). Each participant offered four exemplars per category in a round-robin fashion under the admonition to avoid repeating another person's responses. In the two subsequent tasks, participants attempted to recall the exemplars that they had offered (recall-own task) and to generate new exemplars that had not been offered earlier by themselves or by their fellow group members (generate-new task). Because of the admonition instruction, plagiarisms were counted in the initial generation and generate-new tasks whenever a participant offered an exemplar that had already been given. In the recall-own task, plagiarisms occurred whenever a participant claimed to have generated an exemplar that had been offered by another group member. In all three tasks, the proportion of plagiarized responses was substantial and varied with manipulations such as task difficulty (e.g.,

We wish to thank Leslie Browning, Brandon Gibb, and Anne Kovach for their dedicated help in collecting the data. Appreciation is expressed to Marty Bink, Jason Hicks, and Andy Leynes for their helpful comments on an earlier draft. This work was supported by a Sigma-Xi Grants-in-Aid. Correspondence should be addressed to J. D. Landau, Department of Psychology, University of Georgia, Athens, GA 306023013 (e-mail: josh@meme.psy.uga.edu). orthographic vs. semantic category generation) and retention interval (Brown \& Halliday, 1991).

In the second paradigm, Marsh and his colleagues (Marsh \& Bower, 1993; Marsh \& Landau, 1995) used these same three tasks in the context of a Boggle puzzle task. A Boggle puzzle consists of a $4 \times 4$ square matrix of 16 letters in which words can be found by stringing adjacent letters together (see the Appendix for an example). Like initial generation in the category generation paradigm, the participant generated $25 \%$ of the responses and another source (in this case a computer) generated the remaining $75 \%$ of the responses. Following initial generation, participants attempted to recall their solutions and to generate new solutions that had not been produced earlier. The Boggle paradigm produced much higher rates of plagiarism, which Marsh and Bower argued was a function of the puzzle task's greater ecological validity and the nature of the memory cues that it provided. In the experiments reported in this article, the Boggle puzzle paradigm was used again as a means of testing whether the plagiarism that it elicits can be considered a specific form of a source-monitoring error (Brown \& Halliday, 1991; Jacoby \& Kelley, 1987; Johnson, Hashtroudi, \& Lindsay, 1993; Marsh \& Landau, 1995; Marsh, Landau, \& Hicks, in press). If it is, the incidence of plagiarism should be predictable from the principles of the source-monitoring framework.

In general, source monitoring is the process of remembering where information has originated (Johnson et al., 1993; Johnson \& Raye, 1981; Johnson, Raye, Foley, \& Foley, 1981). According to the source-monitoring framework (see Johnson et al., 1993, for a recent review), successful source monitoring depends on two components: the attributes of the memory representation and the decision processes used to determine that representation's origin. The memorial attributes of a stored representa- 
tion include such information as cognitive operations, sensory/ perceptual details, contextual cues, semantic detail, and affective reactions to the encoding experience. As a guiding principle, whenever the attributes of memory representations are uniquely defined (i.e., highly differentiated), the framework predicts fewer source-monitoring errors. In contrast, when the attributes of a memory overlap with other memories (i.e., they are highly confusable), assigning source is more difficult and the number of source errors should increase.

Although source-monitoring errors can arise from greater overlap among memory representations, they can also occur if the decision processes that inspect these memorial records are biased or inaccurate. In general, decision processes vary in terms of the amount and kind of information that is needed in order to make a source ascription (Dodson \& Johnson, 1996; Multhaup, 1995). In terms of amount, decisions can be made quickly and heuristically or they can be made slowly and deliberately (Johnson et al., 1993). In terms of kind, some situations will demand more perceptual detail (e.g., determining that one has met someone at a party), whereas other situations will require more cognitive operations for one to confidently determine the origin of a memory (e.g., trying to remember where one has encountered some medical advice). Task demands and one's agenda at the time a source-monitoring judgment is required will determine the degree to which additional cognitive processing will be recruited to examine the attributes of the memory. In brief, the application of insufficient or inappropriate decision criteria will cause source-monitoring errors (Dodson \& Johnson, 1996; Johnson et al., 1993; Multhaup, 1995).

The issue of applying appropriate decision processes has important implications for the paradigms of unconscious plagiarism described before. Careful analysis of each of the three plagiarism tasks indicated that the recallown task differs from the two generation tasks in fundamental ways in terms of the decision processes involved. The decision processes associated with the two generation tasks bear some resemblance to old-new recognition in that people generate a puzzle solution and need only to distinguish old items from new items in order to correctly offer a new solution as required by the task. Therefore, identification of old solutions can rely on less differentiated information (e.g., familiarity) as well as less extended reasoning (Dodson \& Johnson, 1996; Johnson et al., 1993). In contrast, the recall-own task might require an old-new decision, but also require determining which of the old items is one's own and which was given by the "partner" (Marsh \& Bower, 1993). This additional decision in the recall-own task is clearly of the same type as those made in standard investigations of the source-monitoring framework in which people are attempting to distinguish between two classes of old items (e.g., Johnson et al., 1981; Zaragoza \& Lane, 1994). If this analysis is correct, successful completion of the recall-own task should require more differentiated information and more systematic decision processes than the two generative tasks, in which a single old-new judgment might suffice. In support of this claim, Marsh and Bower (1993) found that the recall-own task took longer to complete than either of the generation tasks. That finding is also consistent with the fact that source-monitoring judgments require more time to complete than do old-new recognition judgments (Johnson, Kounios, \& Reeder, 1994). Given the preceding discussion, we hypothesized that standard manipulations designed to influence source monitoring should influence performance on the recallown task to a greater degree than the two generation tasks.

To test a similar hypothesis, Marsh and Bower (1993) had participants play Boggle against the computer. In a standard condition, the computer's items appeared on the computer monitor below the puzzle matrix. This condition was compared with one in which only fragments of the solution word were given. People were required to complete the computer's solution by typing in the missing letters. Presumably, having people generate the missing letters for each computer solution should have created more similar cognitive operations between computer- and self-generated solutions. Increasing the similarity of the cognitive operations associated with two sources should have led people in the recall-own task to claim that more computer-generated items were their own. Curiously, and inconsistent with the preceding argument, that result was not obtained: When attempting to recall their own solutions, people in the fragment condition plagiarized the same number of items as did people in the standard condition. Marsh and Bower argued that the circumstances giving rise to cryptomnesia were qualitatively distinct: Cryptomnesia errors could not be wholly subsumed by the source-monitoring framework. Although certain tasks and conditions may support Marsh and Bower's general claim, we find the preceding theoretical analysis of the recall-own task, framed in terms of source monitoring, sufficiently compelling to warrant the present experimentation.

In Experiment 1, we attempted to make the cognitive operations associated with the self-generated and computergenerated solutions more confusable by having participants attempt to guess the computer's solutions during initial generation. Searching the puzzle for the computer's solutions closely resembles the type of search process that people perform when they are searching for one of their own solutions. This type of processing should make the computer's solutions less differentiated from the self-generated items, which, in turn, should increase the proportion of recall-own plagiarisms. Alternatively, it is possible that generating the computer's solutions might result in deeper processing, which would lead to better memory for those items and consequently less plagiarism. For example, Marsh and Bower (1993) found that deeper processing during initial generation led to fewer plagiarisms than did a shallow encoding task. 
Other attributes of the memory representation contribute to source monitoring, such as the perceptual/ sensory qualities and the contextual details associated with a particular source. For example, trying to determine who generated an idea or a concept is much easier when the two sources are a male and a female than when they are both female (Johnson, Nolde, \& De Leonardis, 1996; Lindsay, Johnson, \& Kwon, 1991). In this case, better source memory for the information was supported by the perceptual detail of hearing it in distinctly different voices. In Experiment 2, the level of perceptual differentiation associated with the computer-generated and self-generated solutions was manipulated. In one condition, the experimenter read the computer's solutions, and in another, the participants read the computer's solutions, directly from the computer screen. Presumably, more perceptual and contextual details are associated with memories for these puzzle solutions when another person reads the solutions aloud than when they are presented on a relatively contextually impoverished computer screen. On the one hand, increasing the perceptual and contextual distinctiveness of the two sources should lead to fewer recall-own plagiarisms for the people who play Boggle with a partner. On the other hand, it is possible that the level of plagiarism might increase for people who play Boggle with the computer because of the sensory match effect (Snodgrass, Hirshman, \& Fan, 1996). The sensory match effect refers to the finding that memory for an item is better when that item is presented in the same sensory form at study and test. Thus, participants who study the computer's solutions on the computer screen might have better memory for those items, which would allow them to avoid unconsciously plagiarizing them during the recall-own task.

In summary, the following two experiments were manipulations designed to make the self- and other-generated solutions either highly confusable (Experiment 1) or highly distinguishable (Experiment 2). When the memorial attributes associated with the two sources are easily confused, the level of plagiarism in the recall-own task should increase. In contrast, when the sources are sufficiently differentiated, the number of recall-own plagiarisms should decrease. Although a strict analysis predicts that there should not be any consequent effect on the two generation tasks, these manipulations are also ones that might affect memorability of the items as a whole. Thus, there might be some marginal impact on performance in the generation tasks, but it should be attenuated as compared with the recall-own task.

\section{EXPERIMENT 1}

In this experiment, participants assigned to a generate-reveal condition were shown the computer's solutions letter by letter. As each letter was added, participants were asked to guess which word was being generated. In contrast, participants in the read-intact condition simply read each of the computer's solutions, which were offered in their intact form. On the basis of the preceding analy- sis, if plagiarism errors in the recall-own task follow the general principles of the source-monitoring framework, an increase in the number of recall-own plagiarisms should occur, Marsh and Bower's (1993) original treatment notwithstanding.

\section{Method}

Participants. Forty-two undergraduates from the University of Georgia volunteered and received course credit for their participation. Participants were randomly assigned to the generate-reveal condition or to the read-intact condition. Each participant was tested individually in sessions that lasted approximately $1 \mathrm{~h}$.

Materials and Procedure. Four Boggle puzzles were taken from those used by Marsh and Bower (1993). The puzzles consisted of 16 letters arranged in a $4 \times 4$ matrix and were presented in the center of a computer monitor. The procedure generally follows Marsh and Bower's (Experiment 1) delayed condition. At the beginning of the experimental session, participants in both conditions completed initial generation. In this task, participants alternated with the computer to generate puzzle solutions. Each participant received detailed instructions regarding the rules for generating solutions. Each valid solution had to contain at least three adjoining letters that could be connected horizontally, vertically, or diagonally. However, no letter in the matrix could be used twice in the same word. Moreover, proper nouns were not allowed, and once the root form of a word had been used, additional words could not be created by simply adding a prefix or suffix. For example, if a participant generated the word cat, he/she could not offer cats later in the puzzle. Of course, participants were admonished not to copy solutions that had been used earlier.

Initial generation consisted of four rounds per puzzle and each round was conducted as follows: First the computer offered three solutions, which were the odd-numbered items of highest frequency from the Marsh and Bower Boggle norms. Following the three computer solutions, the participant had to find and offer a new solution. Therefore, by the end of each puzzle, the participant had generated 4 solutions and the computer had offered 12 solutions. The computer software was written so that it contained a safeguard preventing the computer from plagiarizing the solutions offered by the participant.

For each computer solution, people in the read-intact condition pressed a key to verify that they had found it in the puzzle. In contrast, rather than simply reading the computer's solutions, people in the generate-reveal condition were gradually shown each computer solution letter by letter and asked to guess aloud as each additional letter was added. Regardless of whether a guess could be made, participants could reveal the next letter by pressing the space bar. For example, the computer might generate the letter $s$. The participant was instructed to look at the puzzle and to guess which word the computer was generating. Following each guess, the participant revealed the next letter in the word (e.g., s, st, sta, stal, stall) by pressing the space bar. Participants were encouraged to guess with each additional letter. Regardless of when the solution was figured out, people were required to reveal the remaining letters in order to equate presentation duration across participants. Once the entire word was revealed, the participant read it aloud, confirmed that it was in the puzzle, and then proceeded to the next solution.

Once initial generation has been completed for all four puzzles, participants were shown each puzzle and asked to (1) recall and type in the four solutions they had contributed from that puzzle (recall-own task) and (2) type in four new solutions that had not been offered earlier (generate-new task). All tasks were performed on the computer with the relevant puzzle displayed in the center of the screen. Participants were always required to give four responses to each of the three tasks before being allowed to continue.

\section{Results and Discussion}

In all analyses, the significance level was set at .05 . Table 1 sets forth the proportion of incorrect responses by task and condition. Discussion of the results follows the same sequence as testing. 
Table 1

Proportion of Incorrect Responses $\times$ Task and Condition for Experiments 1 and 2

\begin{tabular}{|c|c|c|c|c|c|c|c|c|}
\hline \multirow[b]{4}{*}{ Task } & \multicolumn{8}{|c|}{ Condition } \\
\hline & \multicolumn{4}{|c|}{ Experiment 1} & \multicolumn{4}{|c|}{ Experiment 2} \\
\hline & \multicolumn{2}{|c|}{ Intact-Read } & \multicolumn{2}{|c|}{ Generate-Reveal } & \multicolumn{2}{|c|}{ Computer Partner } & \multicolumn{2}{|c|}{ Human Partner } \\
\hline & $M$ & $S E$ & $M$ & $S E$ & $M$ & $S E$ & $M$ & $S E$ \\
\hline Generation plagiarism & .18 & .02 & .13 & .03 & .17 & .02 & .13 & .02 \\
\hline Recall-own plagiarism & .46 & .03 & .56 & .04 & .50 & .03 & .42 & .02 \\
\hline Recall-own errors & .18 & .02 & .21 & .02 & .21 & .02 & .18 & .02 \\
\hline Generate new & .38 & .03 & .34 & .03 & .42 & .04 & .36 & .03 \\
\hline Other plagiarism & .28 & .02 & .25 & .02 & .29 & .03 & .28 & .03 \\
\hline Self-plagiarism & .10 & .02 & .09 & .02 & .13 & .02 & .08 & .02 \\
\hline
\end{tabular}

In the initial generation task, participants generated four solutions per puzzle. A plagiarism error was counted whenever a participant repeated a solution that had been previously generated. As can be seen in the left half of Table 1, revealing the computer's items led to slightly fewer plagiarisms, but this difference was not significant $[F(1,40)=$ $\left.1.93, M S_{\mathrm{e}}=.01, p=.17\right]$. In the recall-own task, with the puzzle in front of them, participants attempted to recall the solutions that they had contributed during initial generation. This task yields two types of incorrect responses. Participants could either claim to recall an item that had actually been generated earlier by the computer (i.e., a recall-own plagiarism) or claim to recall an item that had not been offered at all during initial generation (i.e., recallown error, or intrusion). As predicted by the source-monitoring framework and our analysis of this paradigm, revealing the computer's solutions increased the proportion of recall-own plagiarisms $\left[F(1,40)=4.23, M S_{\mathrm{e}}=.03\right]$. The number of recall-own errors did not differ $[F(1,40)=$ $\left.1.02, M S_{\mathrm{e}}=.01, p=.31\right]$. More importantly, comparison of the two errors that could be made in the recall-own task demonstrated that people in both conditions were more likely to plagiarize an earlier solution (recall-own plagiarisms) than to offer a response that had not been offered earlier (recall-own errors) $\left[F(1,40)=166.07, M S_{\mathrm{e}}=.01\right]$. This difference is important because it shows that errors due to prior exposure (i.e., priming) exceed intrusions errors (see Brown \& Murphy, 1989).

During the generate-new task, people attempted to offer solutions that had not been offered earlier. In this task, a person could mistakenly regenerate a solution that had been offered by the computer (other plagiarisms) or by themselves (self-plagiarisms). Following Marsh and Bower (1993), we pooled across plagiarism type and analyzed the total amount of plagiarism. As can be seen in Table 1, revealing the solutions during initial generation did not affect the incidence of plagiarism in the generatenew task $\left[F(1,40)=1.36, M S_{\mathrm{e}}=.02, p=.25\right]$.

Revealing the computer's solutions and asking people to guess their identity led to a negligible decrement in plagiarisms in the two generative tasks. More importantly, as predicted by the source-monitoring framework, revealing the computer's solutions increased the number of plagiarisms in the recall-own task. Guessing the computer's solutions made these solutions more similar to self-generated items, and when asked to recall their own contribution, people confused the source of computer-generated with their self-generated solutions.

\section{EXPERIMENT 2}

In contrast to Experiment 1, in which the number of recall-own plagiarisms was increased, the goal of Experiment 2 was to decrease the number of plagiarisms in that task by making the computer- and self-generated solutions more easily distinguishable. Recall that we proposed that the recall-own task requires extended source monitoring and that the two generative tasks can rely on mere recognition. If this is true, increasing the distinctiveness of perceptual qualities and the contextual attributes associated with the "other-generated" information should reduce source errors in the recall-own task but leave plagiarism in the generative tasks fairly constant. During initial generation, half of the participants played Boggle with a human partner and the remaining participants played the game with a computer partner. We predicted that the human partner condition would improve source monitoring and reduce recall-own plagiarisms.

\section{Method}

Participants. Forty undergraduates from the University of Georgia, none of whom had participated in Experiment 1, volunteered and received course credit for their participation. Participants were randomly assigned to the computer- or human-partner condition. Each participant was tested individually in sessions that lasted approximately $1 \mathrm{~h}$.

Materials and Procedure. The materials and procedure were identical to those used in the read-intact condition of Experiment 1, with one modification. During initial generation, participants in the computer-partner condition sat in front of the computer monitor and played Boggle with the computer. Each computer-generated solution was presented directly below the puzzle. In contrast, people in the humanpartner condition sat next to the computer, where they could not see the computer monitor. These participants were given a paper version of each puzzle. Rather than read their partner's solution from the computer screen, their human partner pronounced each computer-generated solution aloud. Participants nevertheless had to signal that they had found the word in the puzzle, as in the computer-partner condition. As in Experiment 1, following initial generation, participants completed the recall-own and generate-new tasks for each puzzle on the computer.

\section{Results and Discussion}

As can be seen in Table 1, during initial generation, people who played Boggle with a human partner plagia- 
rized slightly fewer items than did people who played with a computer partner. However, this difference was not significant $\left[F(1,38)=1.58, M S_{\mathrm{e}}=.01, p=.22\right]$. Playing Boggle with a human partner was predicted to decrease the number of recall-own plagiarisms because there were more perceptual and contextual cues associated with the responses offered by the human partner. This prediction was confirmed $\left[F(1,38)=5.28, M S_{\mathrm{e}}=\right.$ $.01]$. There were, however, no differences in the number of recall-own errors $\left[F(1,38)=1.86, M S_{\mathrm{e}}=.006, p=\right.$ .18]. As in Experiment 1, participants were sensitive to the information encountered during initial generation, as evidenced by a higher proportion of recall-own plagiarisms than recall-own intrusions $[F(1,38)=292.76$, $\left.M S_{\mathrm{e}}=.001\right]$. During the generate-new task, people in each condition plagiarized the same number of solutions $\left[F(1,38)=1.6, M S_{\mathrm{e}}=.03, p=.21\right]$.

As predicted, playing Boggle with a human partner created more differentiated memories, which decreased the amount of plagiarism in the recall-own task.

\section{GENERAL DISCUSSION}

Collectively, these two experiments were designed to assess the role that source monitoring might play in laboratory analogues of unconscious plagiarism. As predicted by the source-nionitoring framework, and our analysis of those analogues, the number of recall-own plagiarisms increased when the computer-generated solutions were made less distinguishable from the self-generated solutions (Experiment 1) and decreased when the two sources were made more distinguishable (Experiment 2 ). In contrast, the two generation tasks were relatively immune to these manipulations, although in each of the two experiments small differences were observed that are attributable to overall encoding differences

As noted, a careful analysis of the three plagiarism tasks revealed that the recall-own task differs from the two generation tasks in terms of the decision processes it requires. The two generation tasks (i.e., the initial generation and generate-new tasks) require finding a new solution. Under these circumstances, people generate a candidate and do not need to engage in extended source monitoring. Rather, they need only to determine whether the item was given before, a judgment akin to an old-new recognition judgment. Although manipulations predicted to affect source monitoring could affect recognition, as Johnson et al (1994) have remarked, recognition is generally a much faster process that does not involve detailed inspection of memorial records. Consistent with this view, the present findings suggest that the generative tasks were less affected by manipulations known to affect source monitoring.

Why do plagiarisms occur in these tasks? Plagiarism might arise by one of several routes, either through errors in recognition or because people fail to apply appropriately stringent enough decision criteria. We have argued that there may not be as much source monitoring going on in these generative tasks as in the recall-own task. This does not exclude the possibility that we have raised elsewhere (Marsh, Landau, \& Hicks, 1996 , in press) that people who are busily engaged in generating a new solution may not adequately monitor the source of each candidate (e.g., Allen \& Jacoby, 1990; Jacoby \& Kelley, 1987). Our main point is that, in the two established paradigms used to study cryptomnesia, extended source monitoring may be localized to one third of the entire experimental sequence. Further, this position does not deny that recognition judgments may be a simple form of source monitoring.

Due to the extended use of source monitoring during the recall-own task, plagiarism levels were sensitive to factors related to the attributes of the memory representations rather than the application of insufficient decision criteria. According to our analysis, the recall-own task is conceptually similar to traditional source-monitoring tasks in which the determination of source is the primary task. In standard source identi- fication tasks, participants encode information from two different sources (e.g., Source A and Source B). At test, these items are presented along with some new items, and people are asked to decide the origin of the item (A, B, or new). On these sorts of tests, people are probably applying about as stringent decision criteria as they can. Under these circumstances, attributes of memorial records will be closely inspected, and when they are manipulated under various tasks and conditions, will yield better or worse source monitoring, depending on how differentiated the two classes of old items are made. If the recall-own task required the same sort of source monitoring to distinguish among old items in service of offering one's old solutions, then more plagiarism should be observed when the computer's items are more like one's own, as occurred in Experiment 1. Similarly, when the attributes of one's items are made more different, plagiarism should decrease, as in Experiment 2.

The results from Experiment 1 stand in stark contrast to Marsh and Bower's (1993, Experiment 2B) failure to find an increase in recall-own plagiarisms when the cognitive operations associated with the self- and computer-generated solutions were manipulated to be more similar. Recall that Marsh and Bower had participants fill in the missing letters for the solutions generated by the computer. The simple explanation is that filling in fragments for the computer's solutions was too weak a manipulation. Filling in the fragments did not lead participants to engage in the same type of processing that occurs when they are generating their own solutions. In contrast, guessing the computer's solutions required people to engage in the same cognitive operations for computer solutions and self-generated items.

We do wish to raise, and dismiss, one potential criticism of the guessing manipulation. In the process of guessing, people occasionally guessed the wrong puzzle solution until a sufficient number of letters had been revealed. 1 Thus, people in that condition might have been exposed to a larger number of solutions overall. In practice, participants often used the incorrect guess as their own next contribution (approximately $27 \%$ of the trials). First, this is just the sort of overlap in cognitive operations that we had hoped the guessing manipulation would generate. By this view, the criticism is actually a restatement of the specific manipulation, only in its stronger form. Second, Marsh and Landau (1995) exposed people to the 32 normatively most frequent Boggle solutions in each puzzle just prior to the recall-own and generate-new tasks. That manipulation had no consequent impact on the plagiarism in either subsequent task. Thus, what at first may appear to be a criticism is unlikely to affect, except in a positive way, the claims we have made.

The results from these two experiments are consistent with other findings as well. In one series of experiments (Marsh et al., in press), groups of participants generated solutions to everyday problems (e.g.. "How can we improve the university?"). After a 1-week delay, participants returned to the laboratory and were asked to individually generate four new ideas that had not been offered during the previous session (i.e., a generate-new task). After these new ideas were collected. each participant was given a source-monitoring test in which the ideas from the previous week were mixed with an equal number of new ideas. For each idea, people were asked to indicate if it was theirs, someone else's, or a new item. Although there were relatively few plagiarisms on the source-monitoring test, there were a substantial number of plagiarisms elicited in the generate-new task. When a person's goal was to ascribe source, sufficiently stringent decision criteria were recruited in order to avoid plagiarism. Conversely, when the goal of a task was to create a new idea or offer a new puzzle solution, people did not seem to devote sufficient attention to the source of their offerings. In other words, people did not use a lot of the information they had available during the generation of a new idea. Consistent with the source-monitoring framework, this is strong evidence that people's decision processes are wholly determined by the demands of the task.

The importance of cryptomnesia transcends its theoretical implications concerning erroneous claims about one's memory. The phenomenon raises practical issues regarding copyright infringement and the ownership of intellectual information. A recent example emphasizes the importance of identifying the factors and situations that influence the incidence of unconscious plagiarism. A political writer for the $N e \mathrm{w}^{\prime} \boldsymbol{e}^{-}$ public admitted that she copied another writer's material when preparing an article profiling former presidential candidate Steven Forbes (Ahrens, 1995). According to her, she inadvertently plagiarized sen- 
tences from another article because she accidently confused her research materials with another author's piece, which had appeared in the National Journal. Apparently, as she was preparing the article, she simultaneously used her word processor, which contained notes about the topic, and an on-line database of articles printed in other publications. Because she failed to clearly label the two sources on her computer screen, this caused her to confuse material from the database with notes she had prepared earlier.

The results that we have reported from the Boggle paradigm suggest that this variety of plagiarism might arise because the writer applied relatively lax decision criteria. Writing an article or creating new technological devices is akin to the generate-new task, in which the primary goal is the production of novel work. The primary goal is not to cautiously monitor sources. In fact, source monitoring at the early stages of a project might obstruct progress because it might inhibit the generation of ideas that are similar (but sufficiently different) from older ideas. Rather than disrupt the creative process, a writer might benefit from scrutinizing the source of each idea upon completion of a piece of work. In this way, the creative process could proceed unimpeded, and the writer could later apply the more stringent type of decision processes that are used by participants during the recall-own task in order to avoid plagiarizing information.

\section{Conclusions}

In analyzing the general procedure used in two of the established laboratory analogues of cryptomnesia, it was hypothesized that correctly generating new exemplars might require no more cognitive processing than that used on a recognition test (i.e., distinguishing old from new items). In contrast, correctly offering one's old items required that they be distinguished from another source's old items. This second discrimination seemed to require source monitoring. Consistent with the predictions of the source-monitoring framework, changes in memorial attributes affected plagiarism in the recall-own task, but not in generative activities. Plagiarisms on generative activities may reflect incorrect recognition decisions or the inadequate application of source-monitoring decision criteria because cognitive processing is diverted toward generating something novel.

\section{REFERENCES}

AHRENS, F. (1995, July 18). A writer's repetitive stress: New Republic admits phrases were copied. Washington Post, pp. C1, C4.

ALLEN, S. W., \& JACOBY, L. L. (1990). Reinstating study context produces unconscious influences of memory. Memory \& Cognition, 18 270-278.

Brown, A. S., \& Halliday, H. E. (1991). Cryptomnesia and source memory difficulties. American Journal of Psychology, 104, 475-490.

Brown, A. S., \& MURPHY, D. R. (1989). Cryptomnesia: Delineating inadvertent plagiarism. Journal of Experimental Psychology: Learning, Memory, \& Cognition, 15, 432-442.

Dodson, C. S., \& Johnson, M. K. (1996). Some problems with the process-dissociation approach to memory. Journal of Experimental Psychology: General, 125, 181-194.

JACOBY, L. L., \& KelleY, C. M. (1987). Unconscious influences of memory for a prior event. Personality \& Social Psychology Bulletin, $13,314-336$.

Johnson, M. K., Hashtroudi, S., \& Lindsay, D. S. (1993). Source monitoring. Psychological Bulletin, 114, 3-28.

Johnson, M. K., Kounios, J., \& ReEder, J. A. (1994). Time-course studies of reality monitoring and recognition. Journal of Experimental Psychology: Learning, Memory, \& Cognition, 20, 1409-1419.

Johnson, M. K., Nolde, S. F., \& DE Leonardis, D. M. (1996). Emotional focus and source monitoring. Journal of Memory \& Language, 35, 135-156.

Johnson, M. K., \& RaYe, C. L. (1981). Reality monitoring. Psychological Review, 88, 67-85.

Johnson, M. K., Raye, C. L., Foley, H. J., \& Foley, M. A. (1981) Cognitive operations and decision bias in reality monitoring. American Journal of Psychology, 94, 37-64.

LindSAY, D. S., Johnson, M. K., \& Kwon, P. (1991). Developmental changes in source monitoring. Journal of Experimental Child Psychology, 52, 297-318.

MarSH, R. L., \& Bower, G. H. (1993). Eliciting cryptomnesia: Unconscious plagiarism in a puzzle task. Journal of Experimental Psychology: Learning, Memory, \& Cognition, 19, 673-688.

MARSH, R. L., \& LANDAU, J. D. (1995). Availability in cryptomnesia: Assessing its role in two paradigms of unconscious plagiarism. Journal of Experimental Psychology: Learning, Memory. \& Cognition, 21, 1568-1582.

Marsh, R. L., Landau, J. D., \& Hicks, J. L. (1996). How examples may (and may not) constrain creativity. Memory \& Cognition, 24, 669-680.

MARSh, R. L., LANDAU, J. D., \& Hicks, J. L. (in press). Contribution of inadequate source monitoring to unconscious plagiarism during idea generation. Journal of Experimental Psychology: Learning, Memory, \& Cognition.

Multhaup, K. S. (1995). Aging, source, and decision criteria: When falsefame errors do and do not occur. Psychology \& Aging, 10, 492-497.

SNODgrass, J. G., Hirshman, E., \& FAN, J. (1996). The sensory match effect in recognition memory: Perceptual fluency or episodic trace? Memory \& Cognition, 24, 367-383.

TAYLOR, F. K. (1965). Cryptomnesia and plagiarism. British Journal of Psychiatry, 111, 1111-1118

ZaraGoza, M. S., \& LANE, S. M. (1994). Source misattributions and the suggestibility of eyewitness memory. Journal of Experimental Psychology: Learning, Memory, \& Cognition, 20, 934-945.

\section{NOTE}

1. Participants in the generate-reveal condition produced less than one incorrect guess per trial (.68).

\section{APPENDIX}

An example of a Boggle puzzle (Marsh \& Bower, 1993):

$\begin{array}{cccc}\text { S } & \text { T } & \text { E } & \text { Y } \\ \text { C } & \text { A } & \text { O } & \text { S } \\ \text { G } & \text { L } & \text { I } & \text { N } \\ \text { A } & \text { N } & \text { L } & \text { D }\end{array}$

Potential solutions: tall, lid, sin, etc.

(Manuscript received August 20, 1996; revision accepted for publication December $27,1996$. 